\title{
Condiciones socioambientales y prevalencia de enfermedades de la piel en un asentamiento urbano precario de Asunción (Paraguay)
}

\author{
Socio-Environmental Conditions and \\ Prevalence of Skin Diseases in a Precarious \\ Urban Settlement in Asunción (Paraguay) \\ Condições socioambientais e prevalência de \\ dermatoses em um assentamento urbano \\ precário em Assunção (Paraguai) \\ Iván Merino, $\mathrm{PhD}(\mathrm{c})^{1}$ \\ Paloma Fernández, MD, Esp. ${ }^{2}$ \\ Paloma Rodero, $\mathrm{PhD}(\mathrm{c})^{3 *}$
}

Recibido: 29 de julio de 2021 • Aceptado: 8 de noviembre de 2021

Doi: https://doi.org/10.12804/revistas.urosario.edu.co/revsalud/a.10915

Para citar este artículo: Merino I, Fernández P, Rodero P. Condiciones socioambientales y prevalencia de enfermedades de la piel en un asentamiento urbano precario de Asunción (Paraguay). Rev Cienc Salud. 2021;20(1):1-23. https://doi.org/10.12804/revistas.urosario.edu. co/revsalud/a.10915

1 Programa Doctorado en Antropología, Universidad Nacional de Educación a Distancia (España) y Universidad de Buenos Aires (Argentina). Dirección de Posgrado e Investigación, Facultad de Filosofía y Ciencias Humanas, Universidad Católica "Nuestra Señora de la Asunción” (Paraguay).

2 Servicio de Dermatología, Hospital Central, Instituto de Previsión Social (Paraguay). Facultad de Ciencias de la Salud, Universidad Católica "Nuestra Señora de la Asunción” (Paraguay).

3 Programa de doctorado en Antropología, Universidad Nacional de Educación a Distancia (España) y Universidad de Buenos Aires (Argentina). Dirección de Postgrado e Investigación, Facultad de Filosofía y Ciencias Humanas, Universidad Católica "Nuestra Señora de la Asunción” (Paraguay).

Iván Merino, ORCID: https://orcid.org/0000-0003-4500-1181

Paloma Fernández, orciD: https://orcid.org/0000-0002-9491-2275

Paloma Rodero, oRCID: https://orcid.org/0000-0003-0650-6435

Autora de correspondencia: palomarodero@yahoo.es 


\section{Resumen}

Introducción: se buscó analizar la prevalencia de enfermedades dermatológicas de la población de un asentamiento urbano precario próximo al vertedero municipal de Asunción (Paraguay), atendiendo a la exposición de las condiciones socioambientales. Materiales y métodos: estudio descriptivo y transversal con una muestra no probabilística intencional que incluyó a 77 personas de entre 5 meses y 76 años. Resultados: en el momento del examen físico, el $96.2 \%$ de las personas presentó, al menos, una lesión cutánea, y el 79.2\%, una o más enfermedades infecciosas de piel; sin embargo, el $77.9 \%$ no había recurrido a consulta médica. Las más sobresalientes fueron las micosis (35.1\%), las bacterianas (35.1\%) y las ectoparasitosis (27.3\%). Se identificó que la prevalencia de enfermedades bacterianas de piel apunta a una posible mayor exposición a condiciones adversas como: un clima subtropical de altas temperaturas y húmedo, una alta relación de convivientes en el hogar por dormitorio, problemas de provisión de agua y suelos de arena en los hogares, así como un sistema de evacuación de excretas deficiente, la presencia de animales con escasa vigilancia sanitaria, la proximidad a arroyos y cauces al aire libre depositarios de materia cloacal y el impacto recurrente de raudales los días de intensa lluvia. Conclusión: los resultados sugieren que la alta prevalencia de enfermedades cutáneas infecciosas podría relacionarse con una mayor exposición a condiciones socioambientales locales adversas, que son factores relevantes por tener en cuenta para mejorar la atención a la salud de las enfermedades dermatológicas de la población urbana que habita en un asentamiento urbano precario.

Palabras clave: condiciones socioeconómicas; salud ambiental; asentamiento informal; enfermedades de piel; Paraguay.

\section{Abstract}

Introduction: This study analyzes the prevalence of dermatological diseases in the population of a precarious urban settlement near the municipal landfill of Asunción (Paraguay), in relation to exposure to socio-environmental conditions. Materials and methods: descriptive, cross-sectional study with a non-probabilistic purposive sample that included 77 people aged between 5 months and 76 years. Results: at the time of physical examination, $96.2 \%$ of the people presented at least one skin lesion and $79.2 \%$ one or more infectious skin diseases, but $77.9 \%$ had not consulted a doctor. The most prominent were mycoses (35.1\%), bacterial (35.1\%) and ectoparasitoses (27.3\%). It was identified that the prevalence of bacterial skin diseases points to possible increased exposure to adverse conditions such as: a subtropical climate with high temperatures and humidity, a high ratio of people living in the household per bedroom, water supply problems and sandy soils in homes, as well as a deficient excreta disposal system, the presence of animals with poor sanitary surveillance, proximity to streams and open-air waterways that deposit sewage and the recurrent impact of floods on days of heavy rainfall. Conclusion: the results suggest that the high prevalence of infectious skin diseases could be related to increased exposure to adverse local socio-environmental conditions, which are relevant factors to be taken into to improve the health care of skin diseases in the urban population living in a slum settlement.

Keywords: socio-economic conditions; environmental health; informal settlement; skin diseases; Paraguay.

\section{Resumo}

Introdução: este estudo analisa a prevalência de doenças dermatológicas na população de um assentamento urbano precário próximo ao aterro sanitário municipal de Asunción (Paraguai) em relação à exposição às condições socioambientais. Materiais e métodos: estudo descritivo, transversal, com uma amostra não-probabilística de propósito que incluiu 77 pessoas com idade entre 5 meses e 76 anos. Resultados: no momento do exame físico, $96,2 \%$ das pessoas tinham pelo menos uma lesão de pele e $79,2 \%$ tinham uma ou mais doenças infecciosas de pele, mas 77,9\% não tinham consultado um médico. 
As mais importantes foram micoses (35,1\%), bacterianas (35,1\%) e ectoparasitoses (27,3\%). A prevalência de doenças bacterianas da pele foi identificada como apontando para um possível aumento da exposição a condições adversas tais como: um clima subtropical com altas temperaturas e umidade, uma alta proporção de pessoas vivendo na casa por quarto, problemas de abastecimento de água e solos arenosos nas habitações, bem como um sistema de eliminação de excrementos deficiente, a presença de animais com vigilância sanitária deficiente, proximidade a riachos e cursos de água abertos que depositam águas residuais, e o impacto recorrente de enchentes em dias de chuvas fortes. Conclusão: Os resultados sugerem que a alta prevalência de doenças infecciosas de pele pode estar relacionada ao aumento da exposição a condições sócio-ambientais locais adversas, que são fatores relevantes a serem considerados para melhorar os cuidados com doenças de pele na população urbana que vive em uma favela.

Palavras-chave: condições socioeconômicas; saúde ambiental; assentamento informal; doenças de pele; Paraguai.

\section{Introducción}

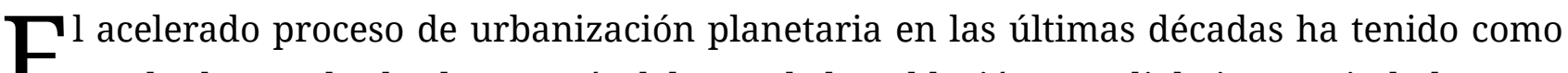
Eresultado que desde el 2007 más del 50\% de la población mundial viva en ciudades. Una tendencia que en muchas urbes ha generado severas disparidades en las condiciones de vida y salud de sus habitantes. En el Sur Global, ha dado lugar particularmente a amplias áreas urbanas de asentamientos precarios con evidentes carencias en condiciones básicas de vida (1).

Esta población urbana se distingue por un sostenimiento de la vida por debajo del estándar socioeconómico medio local y por convivir en una situación de mayor desventaja social, en áreas con un acceso limitado a atención médica, escuelas, zonas recreativas seguras y servicios básicos deficientes, como la falta de recolección de basuras, agua segura o desagüe adecuado de excretas. Además, muchos de estos asentamientos se erigen en territorios vulnerables a desastres naturales (inundaciones, terremotos o deslizamientos de tierra) en áreas geológicas anegadas, cuya fragilización coadyuva en muchas ocasiones con las condiciones materiales precarias de sus viviendas (2).

Se estima que más de la mitad de la población mundial vive en ciudades y que en torno al $30 \%$ de la población urbana se concentra en asentamientos urbanos precarios. Esta tendencia creciente de urbanización supone uno de los más importantes desafíos en salud pública global, realidad que a pesar de su alta relevancia, hasta el momento ha sido escasamente estudiada (3).

Entre los problemas de salud de las poblaciones desfavorecidas que habitan en los asentamientos urbanos precarios se encuentran las enfermedades dermatológicas. Estas, a pesar de ser todavía poco reconocidas, al igual que una variedad de enfermedades crónicas y comunes (infecciosas y no infecciosas), son frecuentes entre su población, llegando al 
sistema formal de salud, en no pocas ocasiones, en sus estadios tardíos o cuando contraen complicaciones (4-8).

En Asunción, estos barrios urbanos precarios se han ido conformando con mayor intensidad desde la década de los setenta, a lo largo de gran parte de su litoral, en las áreas conocidas comúnmente como los Bañados, que en la actualidad reúnen a la quinta parte de la población de la ciudad, unos 100000 habitantes (9).

Con el propósito de poner en valor el estudio de las enfermedades dermatológicas en un contexto urbano precario, este artículo tiene como objetivo analizar la prevalencia de las enfermedades cutáneas, atendiendo a la exposición de las condiciones sociales, habitacionales y ambientales en un territorio con un importante deterioro socioambiental, de difícil acceso y fuerte estigma social, tras las inundaciones del río Paraguay entre 2014 y 2016.

\section{Materiales y métodos}

— ste estudio se desarrolló en un barrio de la periferia sur de Asunción, que se singulariza Epor la localización del vertedero municipal de la ciudad desde hace más de tres décadas. Según las estadísticas oficiales de necesidades básicas insatisfechas, este barrio de la capital del Paraguay concentra los niveles más altos de densidad poblacional, junto con los más bajos de escolaridad, además de reunir elevados índices de empleo en situación de informalidad entre su población y distinguirse por aglutinar precarias condiciones de vivienda y acceso a servicios básicos (10). Del mismo modo, esta zona urbana se particulariza porque parte de su territorio es impactado regularmente por inundaciones, a consecuencia de voluminosos raudales los días de intensa lluvia que irrumpen por el desborde de los arroyos y por haber sufrido cuatro crecidas extraordinarias del río Paraguay en los últimos años (11).

Se realizó un estudio de carácter descriptivo y transversal con una muestra no probabilística de tipo intencional que incluyó a 77 personas. La selección de la muestra estuvo compuesta por habitantes que viven en cuatro microterritorios de los alrededores del vertedero municipal de la ciudad de Asunción, en un radio inferior a 500 metros de su perímetro.

La muestra tuvo como criterios de inclusión que la persona aceptara un examen físico de piel realizado por una doctora especialista y que se completara: la ficha clínica individual de enfermedades cutáneas encontradas y la ficha social de condiciones socioeconómicas, habitacionales y ambientales del hogar de la persona.

El trabajo de campo se desarrolló entre noviembre de 2016 y junio de 2017 mediante visitas domiciliarias por parte de una dermatóloga y de dos antropólogos sociales residentes en la comunidad. 
Los datos se recolectaron mediante dos instrumentos de registro: una ficha social y una ficha clínica individual. La ficha social permitió recabar información sobre la composición de los convivientes del lugar de residencia, sexo, edad y escolaridad de los diferentes miembros, ocupación e ingresos mensuales, los materiales de construcción de la vivienda, su distribución espacial y condiciones higiénico-sanitarias: el tipo de evacuación de excretas y de eliminación de basuras, la existencia de problemas frecuentes en el suministro de agua corriente, el contacto directo o indirecto con aguas de los efluentes circundantes y la presencia de animales. Así mismo, se cumplimentó una ficha clínica individual, centrada en el registro de enfermedades en la piel identificadas en el examen físico realizado por la especialista dermatóloga, describiendo sus lesiones elementales, morfología, localización, evolución y tratamiento. En la ficha social se incorporó también información sobre la distancia en metros de la ubicación de la vivienda con respecto a los cursos de agua cercanos (arroyos, lagunas y río), al perímetro del vertedero y a los cauces de aguas cloacales a cielo abierto, así como la situación orográfica en relación con el drenaje de los raudales de lluvia en el territorio.

Los datos recolectados se sistematizaron mediante una planilla Microsoft Excel@ y se procesaron con el programa estadístico Iвм ${ }^{\circledR}$ spss ${ }^{\circledR}$ Statistics, versión 24. Para el análisis comparativo de datos, primero, se definieron las frecuencias de las distintas características socioeconómicas, habitacionales y ambientales. Luego, se halló la prevalencia de las distintas patologías dermatológicas encontradas en los exámenes físicos. Después, para explorar la posible relación de las enfermedades de piel según las diferentes condiciones habitacionales y ambientales, se utilizó la prueba de chi cuadrado $\left(\chi^{2}\right)$ de Pearson y la prueba exacta de Fisher (para variables dicotómicas y con frecuencia esperada menor de 5) con un nivel de significancia del 0.05.

En relación con las consideraciones éticas, en este estudio se siguieron estrictamente, durante todo el proceso de la investigación, los principios de confidencialidad, anonimización y respeto a la dignidad de las personas, de acuerdo con la Declaración de Helsinki y los códigos y guías éticas de la American Anthropological Association y la Associação Brasileira de Antropología (12-14). Se aplicó el consentimiento informado oral a cada uno de los participantes, los cuales aceptaron participar voluntariamente, tras la explicación previa del objetivo, finalidad y el fiel compromiso del equipo investigador a seguir los códigos éticos durante la investigación. En el caso de los menores de edad, uno de sus progenitores otorgó el consentimiento y este estuvo presente durante la exploración física realizada por la dermatóloga.

Este trabajo no contó con el aval de un comité de ética, por no estar todavía constituido institucionalmente en la fecha de la investigación en el centro de estudios y en el Consejo Nacional de Ciencia y Tecnología (Conacyt) de Paraguay. 


\section{Resultados}

\section{Condiciones socioeconómicas y habitacionales}

La población incluida en este estudio fue de 77 personas de edades comprendidas entre los 5 meses y los 76 años; un $61.0 \%$ eran mujeres y un $39.0 \%$ eran hombres. Por rangos etarios, los menores de 5 años representaron el $20.8 \%$ de la muestra; los escolares de 5 a 14 años, el $27.3 \%$; los jóvenes y adultos de 15 a 49 años, el 44.2\%, y los participantes de 50 y más años, el $7.7 \%$ (tabla 1$)$.

Tabla 1. Características demográficas, socioeconómicas y habitacionales

\begin{tabular}{|c|c|c|}
\hline Características & $\%$ & $\mathbf{n}$ \\
\hline Total & 100.0 & 77 \\
\hline \multicolumn{3}{|l|}{ Sexo } \\
\hline Mujer & 61.0 & 47 \\
\hline Varón & 39.0 & 30 \\
\hline \multicolumn{3}{|l|}{ Rango etario } \\
\hline Menores de 5 años & 20.8 & 16 \\
\hline Escolares de 5 a 14 años & 27.3 & 21 \\
\hline Jóvenes y adultos de 15 a 49 años & 44.2 & 34 \\
\hline 50 y más años & 7.7 & 6 \\
\hline \multicolumn{3}{|l|}{ Ocupación } \\
\hline Menores de 15 años y estudiantes & 50.6 & 39 \\
\hline Reciclador & 28.6 & 22 \\
\hline Ama de casa & 13.0 & 10 \\
\hline Otras actividades laborales & 5.2 & 4 \\
\hline Jubilados & 2.6 & 2 \\
\hline \multicolumn{3}{|c|}{$\begin{array}{l}\text { Condición socioeconómica según ingresos per cápita en el hogar } \\
\text { (conforme a baremos estatales de la DGEEC) }\end{array}$} \\
\hline Pobre (con menos de 113 usD al mes) & 63.6 & 49 \\
\hline Pobre extremo (con menos de 44 usD al mes) & 31.2 & 24 \\
\hline No pobre (con más de 113 usd al mes) & 5.2 & 4 \\
\hline \multicolumn{3}{|l|}{ Protección social en salud } \\
\hline Sin seguro médico & 100.0 & 77 \\
\hline \multicolumn{3}{|l|}{ Materiales de construcción de la vivienda } \\
\hline Piso de tierra & 62.3 & 48 \\
\hline Pared de terciada & 59.7 & 46 \\
\hline Techo de chapa de zinc o fibrocemento (asbesto) & 94.8 & 73 \\
\hline
\end{tabular}




\section{Características}

$\% \quad \mathbf{n}$

\section{Relación de convivientes por hogar}

$\begin{array}{ll}\text { Media de personas compartiendo hogar } & 7.1\end{array}$

Media de dormitorios por hogar $\quad 2.04$

\begin{tabular}{lll} 
Más de tres personas por dormitorio & 62.3 & 48 \\
\hline Problemas de provisión de agua & 94.8 & 73
\end{tabular}

\section{Eliminación de excretas}

Conexión a la red de alcantarillado público

Pozo negro

$0.0 \quad 0$

Al arroyo o laguna

$9.1 \quad 7$

Hoyo o letrina

$23.4 \quad 18$

No dispone de baño

$62.3 \quad 48$

\section{Eliminación de basuras}

Servicio de recolección municipal

$5.2 \quad 4$

Quema o entierra

$2.5 \quad 2$

Tira a laguna o arroyo

$48.1 \quad 37$

Animales en el hogar

Al menos un perro $66.2 \quad 51$

Animales de corral

$46.8 \quad 36$

\section{Impacto de raudales de lluvia en el hogar}

Sí, entra en la casa

$50.6 \quad 39$

Entra solo en el patio

$10.4 \quad 8$

No entra agua en la vivienda

$39.0 \quad 30$

Desplazamientos por crecida del río Paraguay (2014-2016)

Al menos una vez

$97.4 \quad 75$

Tres y cuatro veces

$64.9 \quad 50$

Nunca

$2.6 \quad 2$

\section{Distancia con respecto a un arroyo}

A menos de 50 metros

$28.6 \quad 22$

Entre 51 y 250 metros

$33.8 \quad 26$

Entre 251 y 500 metros

$5.2 \quad 4$

Más de 500 metros

$32.4 \quad 25$

Distancia con respecto a una laguna interna

A menos de 250 metros

$96.1 \quad 74$

Más de 250 metros

3.9

3

\section{Distancia con respecto al río Paraguay}

\begin{tabular}{lrr} 
A menos de 250 metros & 96.1 & 74 \\
Más de 250 metros & 3.9 & 3 \\
\hline & & Continúa
\end{tabular}




\begin{tabular}{lrr}
\hline \multicolumn{1}{c}{ Características } & \% & n \\
\hline Distancia de la vivienda con el vertedero & & \\
De 0 a 50 metros & 35.1 & 27 \\
De 51 a 250 metros & 57.1 & 44 \\
De 251 a 500 metros & 7.8 & 6 \\
\hline Distancia de la vivienda al cauce cloacal a cielo abierto & & \\
De 0 a 50 metros & 10.4 & 8 \\
De 51 a 250 metros & 64.9 & 50 \\
De 251 a 500 metros & 15.6 & 12 \\
Más de 500 metros & 9.1 & 7 \\
\hline
\end{tabular}

Conforme a indicadores de instituciones estatales nacionales, el nivel socioeconómico de los participantes era en un $63.6 \%$ de pobreza y en un $31.2 \%$ de pobreza extrema. En ningún caso la población adulta del estudio había finalizado la escolaridad secundaria. Entre las personas de 15 y más años, una importante proporción se dedicaba a la actividad del reciclado, otras eran amas de casa o se desempeñaban en distintos oficios (mecánico, despensera, jornalera o costurera) y los jubilados recibían una pequeña ayuda asistencial estatal de aproximadamente 73 dólares mensuales (tabla 1).

El conjunto de participantes, en su mayoría, vivía en casas construidas con materiales precarios, entre los cuales predominaban las paredes tipo “terciada” (lámina fina de madera) (59.7\%), el suelo de arena (62.3\%) y la techumbre de chapas de metal o fibrocemento (94.8\%). En gran parte de los hogares, por cada dormitorio existía una relación de tres o más convivientes (62.3\%). Así mismo, casi en su totalidad manifestaron tener problemas frecuentes de provisión regular de agua (94.8\%) (tabla 1 ).

En los hogares de las personas examinadas, los residuos se eliminaban fundamentalmente en arroyos o lagunas cercanas, se quemaban o se cubrían con escombros en el patio de la casa (97.5\%). Respecto a la evacuación de excretas, en su mayoría (85.7\%) disponía de hoyo o letrina común en el patio de la vivienda, o se desaguaban en un arroyo o laguna próxima. Muchas de las personas convivían al menos con un perro (66.2\%) o compartían espacio de residencia con animales de corral (cerdos, gallinas o ambos) (46.8\%) (tabla 1).

En una alta proporción, la residencia se situaba a menos de 250 metros de uno de los cuatro arroyos que atraviesan el territorio donde desembocan tres de los desagües cloacales de este sector de la ciudad (62.4\%). La gran mayoría (96.1\%), a su vez, vivía a menos de 250 metros de una laguna interna y a menos de un kilómetro del río. Un importante porcentaje manifestó ser afectado a menudo por inundación de agua sucia de raudal (proveniente del desborde de los arroyos adyacentes) en el interior de su vivienda o en su patio los días de intensas lluvias (61.0\%). Entre mayo de 2014 y enero de 2016, gran parte de los participantes se vieron obligados a desplazarse a áreas de refugio, al menos en una ocasión, a causa de 
la inundación de su casa por la crecida extraordinaria del río Paraguay (97.4\%). Durante el mismo periodo, una importante proporción llegó a trasladarse hasta tres y cuatro veces a áreas de refugio (64.9\%) (tabla 1).

\section{Enfermedades dermatológicas}

A lo largo de la investigación se realizó examen físico de piel a 77 personas. Estas presentaron una alta prevalencia de enfermedades dermatológicas: en un $96.2 \%$ de los casos se encontró, al menos, una lesión cutánea. Un 62.4\% tenía al menos dos lesiones dermatológicas, y un 32.5 \%, 3 o más patologías cutáneas. Incluso se llegó a observar hasta 7 lesiones cutáneas en una misma persona. A pesar de estos hallazgos, es destacable que un $77.9 \%$ de la población examinada no había recurrido con anterioridad a consulta médica.

Las mujeres concentraron el $65.7 \%$ de los diagnósticos, en contraste con los hombres, en quienes se identificó el 34.3\% de las patologías. Entre las mujeres, un 36.2 \% tenía al menos tres lesiones de piel. Por rango etario, los menores de 15 años reunieron el $48.1 \%$ de las lesiones cutáneas encontradas.

Por grupos de enfermedad, las dermatosis de carácter infeccioso fueron predominantes y alcanzaron una prevalencia del 79.2\%. Las dermatitis y los eccemas fueron el segundo grupo de mayor prevalencia, presentes en el $42.9 \%$ de las personas examinadas; seguidas de las enfermedades de los anexos cutáneos, en un 9.1\% (tabla 2).

Tabla 2. Prevalencia de las enfermedades dermatológicas $(n=77)$

\begin{tabular}{lcc}
\hline \multicolumn{1}{c}{ Diagnósticos } & \% & n \\
\hline Patologías infecciosas & $\mathbf{7 9 . 2}$ & $\mathbf{6 1}$ \\
Micosis superficiales & $\mathbf{3 5 . 1}$ & $\mathbf{2 7}$ \\
Tiñas & 23.4 & 18 \\
Onicomicosis & 10.4 & 8 \\
Pitiriasis versicolor & 5.2 & 4 \\
\hline Infecciones cutáneas bacterianas & $\mathbf{3 5 . 1}$ & $\mathbf{2 7}$ \\
Forúnculo & 19.5 & 15 \\
Impétigo & 10.4 & 8 \\
Foliculitis & 6.5 & 5 \\
Celulitis & 6.5 & 5 \\
Absceso & 1.3 & 1 \\
\hline Ectoparasitosis & $\mathbf{2 7 . 3}$ & $\mathbf{2 1}$ \\
Escabiosis & 15.6 & 12 \\
Larva migratoria cutánea & 10.4 & 8 \\
Tunga penetrans & 2.6 & 2 \\
\hline
\end{tabular}




\begin{tabular}{|c|c|c|}
\hline Diagnósticos & $\%$ & $\mathbf{n}$ \\
\hline Enfermedades virales & 15.6 & 12 \\
\hline Verrugas víricas & 11.7 & 9 \\
\hline Herpes zóster & 1.3 & 1 \\
\hline Herpes simple & 1.3 & 1 \\
\hline Molusco contagioso & 1.3 & 1 \\
\hline Enfermedad de Hansen & 1.3 & 1 \\
\hline Dermatitis y eccema & 42.9 & 33 \\
\hline Dermatitis seborreica & 18.2 & 14 \\
\hline Pitiriasis alba & 14.3 & 11 \\
\hline Dermatitis atópica & 10.4 & 8 \\
\hline Prurigo & 9.1 & 7 \\
\hline Dermatitis de contacto & 2.6 & 2 \\
\hline Eczema varicoso & 1.3 & 1 \\
\hline Enfermedades de los anexos & 9.1 & 7 \\
\hline Acné & 9.1 & 7 \\
\hline Miliaria rubra & 1.3 & 1 \\
\hline Tumores benignos & 7.8 & 6 \\
\hline Hiperplasias sebáceas & 2.6 & 2 \\
\hline Lipoma & 2.6 & 2 \\
\hline Acrocordones & 1.3 & 1 \\
\hline Dermatofibroma & 1.3 & 1 \\
\hline Quiste pilar & 1.3 & 1 \\
\hline Tumores malignos & 1.3 & 1 \\
\hline Carcinoma basocelular & 1.3 & 1 \\
\hline Trastornos de la pigmentación & 9.1 & 7 \\
\hline Melasma & 7.8 & 6 \\
\hline Vitiligo & 1.3 & 1 \\
\hline Heridas cortantes & 7.8 & 6 \\
\hline Úlceras cutáneas & 3.9 & 3 \\
\hline Quemaduras de segundo grado & 2.6 & 2 \\
\hline Otros & 6.5 & 5 \\
\hline Xerosis cutis & 2.6 & 2 \\
\hline Esclerosis sistémica & 1.3 & 1 \\
\hline Manchas café con leche & 1.3 & 1 \\
\hline Alopecia traccional & 1.3 & 1 \\
\hline
\end{tabular}

Según la patología, las dermatofitosis o tiñas fueron las de mayor prevalencia. Afectaban a un $23.4 \%$ de las personas examinadas, sobre todo a las mujeres, las cuales reunieron el 
72.2\% de las lesiones identificadas. Del conjunto de las dermatofitosis halladas, estas tuvieron diferentes localizaciones: el 38.1 \% fueron tiñas de los pies; el 19.1\%, tiñas del cuerpo; el 9.5\%, tiñas de las manos; el 14.3\%, tiñas de la cabeza (todas ellas en niños); el 9.5\%, tiñas inguinales, y el $9.5 \%$, tiñas faciales. En segundo lugar, la patología más prevalente fue el forúnculo, presente en un $19.5 \%$ de las personas examinadas, seguida de la dermatitis seborreica, en un $18.2 \%$; la escabiosis, en un $15.6 \%$, y la pitiriasis alba, en un $14.3 \%$ (tabla 2 ).

Por grupo de edad, cabe destacar el impétigo dentro de las infecciones de la piel identificadas como más predominantes en los niños menores de 5 años, quienes acumularon el $62.5 \%$ de los casos; aunque también se identificó entre las personas más ancianas. Por su parte, el $61.1 \%$ de las tiñas y la totalidad de onicomicosis fueron encontradas en personas de 15 y más años.

Por otro lado, el análisis comparativo de la exposición a condiciones habitacionales y ambientales según patologías de la piel de carácter infeccioso sugirió una posible relación con un número de convivientes mayor a tres personas por dormitorio, fundamentalmente, en personas que presentaron forunculosis y, al menos, una infección en la piel de origen bacteriano, las cuales reportaron en su totalidad problemas de provisión de agua en sus hogares.

De igual modo, se constató una posible relación entre vivir en casas con suelos de arena, con perros y a menos de 250 metros de un cauce cloacal a cielo abierto o un arroyo con presentar alguna enfermedad cutánea de etiología bacteriana o forunculosis. Adicionalmente, la mayoría de las personas con forunculosis eran afectadas por la entrada de agua sucia de raudal cuando llueve y vivía con algún animal (gallinas, cerdos o perros). Las que presentaron, al menos, una infección cutánea bacteriana se habían desplazado más de una vez a las áreas de refugio por motivo de las crecidas extraordinarias del río Paraguay (entre mayo de 2014 y enero de 2016) (tabla 3). 
Tabla 3. Condiciones habitacionales, ambientales y enfermedades de piel

\begin{tabular}{|c|c|c|c|c|c|c|c|c|c|c|c|c|}
\hline \multirow{3}{*}{$\begin{array}{c}\text { Condiciones habitacionales } \\
\text { y ambientales }\end{array}$} & \multicolumn{4}{|c|}{$\begin{array}{c}\text { Con infección cutá- } \\
\text { nea de origen bac- } \\
\text { teriano }\end{array}$} & \multicolumn{4}{|c|}{$\begin{array}{c}\text { Sin infección cutá- } \\
\text { nea de origen bac- } \\
\text { teriano } \\
\end{array}$} & \multirow{3}{*}{ OR } & \multirow{3}{*}{\multicolumn{2}{|c|}{ IC95\% }} & \multirow[t]{3}{*}{$p^{*}$} \\
\hline & \multicolumn{2}{|l|}{ Sí } & \multicolumn{2}{|c|}{ No } & \multicolumn{2}{|l|}{ Sí } & \multicolumn{2}{|c|}{ No } & & & & \\
\hline & $\%$ & $\mathbf{n}$ & $\%$ & $\mathbf{n}$ & $\%$ & $\mathbf{n}$ & $\%$ & $\mathbf{n}$ & & & & \\
\hline $\begin{array}{l}\text { Vivienda con más de tres personas } \\
\text { por dormitorio }\end{array}$ & 81.5 & 22 & 18.5 & 5 & 52.0 & 26 & 48.0 & 24 & 4.062 & 1.327 & 12.428 & 0.011 \\
\hline $\begin{array}{l}\text { Evacuación de } \\
\text { rior del patio }\end{array}$ & 77.8 & 21 & 22.2 & 6 & 76.0 & 38 & 24.0 & 12 & 1.105 & 0.362 & 3.372 & 0.860 \\
\hline $\begin{array}{l}\text { Eliminación de basuras } \\
\text { rior del patio de la vivie }\end{array}$ & 59.3 & 16 & 40.7 & 11 & 42.0 & 21 & 58.0 & 29 & 2.009 & 0.776 & 5.200 & 0.148 \\
\hline Vivienda con perros & 81.5 & 22 & 18.5 & 5 & 58.0 & 29 & 42.0 & 21 & 3.186 & 1.038 & 9.782 & 0.038 \\
\hline $\begin{array}{l}\text { Vivienda con animal (gallinas, cer- } \\
\text { dos o perro) }\end{array}$ & 59.3 & 16 & 40.7 & 11 & 40.0 & 20 & 60.0 & 30 & 2.182 & 0.841 & 5.662 & 0.106 \\
\hline Vivienda con piso de arena & 88.9 & 24 & 11.1 & 3 & 48.0 & 24 & 52.0 & 26 & 8.667 & 2.310 & 32.516 & 0.000 \\
\hline $\begin{array}{l}\text { Desplazada por crecida del río más } \\
\text { de una vez }\end{array}$ & 100.0 & 27 & 0.0 & 0 & 72.0 & 36 & 28.0 & 14 & a & & & 0.001 \\
\hline $\begin{array}{l}\text { Vivienda a m } \\
\text { arroyo }\end{array}$ & 77.8 & 21 & 22.2 & 6 & 54.0 & 27 & 46.0 & 23 & 2.981 & 1.029 & 8.642 & 0.040 \\
\hline $\begin{array}{l}\text { Vivienda a menos de } 250 \mathrm{~m} \text { de un } \\
\text { cauce cloacal a cielo abierto }\end{array}$ & 96.3 & 26 & 3.7 & 1 & 64.0 & 32 & 36.0 & 18 & 14.625 & 1.829 & 116.96 & 0.002 \\
\hline $\begin{array}{l}\text { Entra agua en la vivienda cuando } \\
\text { llueve }\end{array}$ & 74.1 & 20 & 25.9 & 7 & 54.0 & 27 & 46.0 & 23 & 2.434 & 0.873 & 6.782 & 0.085 \\
\hline $\begin{array}{l}\text { Vivienda a menos de } 50 \mathrm{~m} \text { de una } \\
\text { laguna }\end{array}$ & 33.3 & 9 & 66.7 & 18 & 48.0 & 24 & 52.0 & 26 & 0.542 & 0.205 & 1.434 & 0.215 \\
\hline $\begin{array}{l}\text { Vivienda a menos de } 50 \mathrm{~m} \text { del pe } \\
\text { metro del vertedero }\end{array}$ & 25.9 & 7 & 74.1 & 20 & 40.0 & 20 & 60.0 & 30 & 0.525 & 0.187 & 1.471 & 0.217 \\
\hline \multirow[t]{4}{*}{$\begin{array}{l}\text { Problemas en el suministro de } \\
\text { agua corriente }\end{array}$} & 100.0 & 27 & 0.0 & 0 & 92.0 & 46 & 8.0 & 4 & $\mathrm{a}$ & & & 0.291 \\
\hline & \multicolumn{4}{|c|}{ Con forunculosis } & \multicolumn{4}{|c|}{ Sin forunculosis } & \multirow{3}{*}{ OR } & \multirow{3}{*}{\multicolumn{2}{|c|}{ IC95\% }} & \multirow{3}{*}{$p^{*}$} \\
\hline & \multicolumn{2}{|l|}{ Sí } & \multicolumn{2}{|c|}{ No } & \multicolumn{2}{|l|}{ Sí } & \multicolumn{2}{|c|}{ No } & & & & \\
\hline & $\%$ & $\mathbf{n}$ & $\%$ & $\mathbf{n}$ & $\%$ & $\mathbf{n}$ & $\%$ & $\mathbf{N}$ & & & & \\
\hline $\begin{array}{l}\text { Vivienda con más de tres personas } \\
\text { por dormitorio }\end{array}$ & 86.7 & 13 & 13.3 & 2 & 56.5 & 35 & 43.5 & 27 & 5.014 & 1.042 & 24.128 & 0.030 \\
\hline $\begin{array}{l}\text { Evacuación de excretas en el inte- } \\
\text { rior del patio de la vivienda }\end{array}$ & 73.3 & 11 & 26.7 & 4 & 77.4 & 48 & 22.6 & 14 & a & & & 0.741 \\
\hline $\begin{array}{l}\text { Eliminación de basuras en el inte- } \\
\text { rior del patio de la vivienda }\end{array}$ & 60.0 & 9 & 40.0 & 6 & 45.2 & 28 & 54.8 & 34 & 1.821 & 0.578 & 5.739 & 0.302 \\
\hline Vivienda con perros & 93.3 & 14 & 6.7 & 1 & 59.7 & 37 & 40.3 & 25 & 9.459 & 1.169 & 76.575 & 0.013 \\
\hline $\begin{array}{l}\text { Vivienda con animal (gallinas, cer- } \\
\text { dos o perro) }\end{array}$ & 80.0 & 12 & 20.0 & 3 & 38.7 & 24 & 61.3 & 38 & 6.333 & 1.618 & 24.786 & 0.004 \\
\hline Vivienda con piso de arena & 86.7 & 13 & 13.3 & 2 & 56.5 & 35 & 43.5 & 27 & 5.014 & 1.042 & 24.128 & 0.030 \\
\hline
\end{tabular}




\begin{tabular}{|c|c|c|c|c|c|c|c|c|c|c|c|c|}
\hline & \multicolumn{4}{|c|}{ Con forunculosis } & \multicolumn{4}{|c|}{ Sin forunculosis } & \multirow{3}{*}{ OR } & & & \multirow{3}{*}{$p^{*}$} \\
\hline & \multicolumn{2}{|l|}{ Sí } & \multicolumn{2}{|c|}{ No } & \multicolumn{2}{|c|}{ Sí } & \multicolumn{2}{|c|}{ No } & & \multirow{2}{*}{\multicolumn{2}{|c|}{ IC95\% }} & \\
\hline & $\%$ & $\mathbf{n}$ & $\%$ & $\mathbf{n}$ & $\%$ & $\mathbf{n}$ & $\%$ & $\mathbf{N}$ & & & & \\
\hline $\begin{array}{l}\text { Desplazada por crecida del río más } \\
\text { de una vez }\end{array}$ & 100.0 & 15 & 0.0 & 0 & 77.4 & 48 & 22.6 & 14 & $\mathrm{a}$ & & & 0.059 \\
\hline $\begin{array}{l}\text { Vivienda a menos de } 250 \mathrm{~m} \text { de un } \\
\text { arroyo }\end{array}$ & 100.0 & 15 & 0.0 & 0 & 53.2 & 33 & 46.8 & 29 & 1.879 & 1.488 & 2.373 & 0.001 \\
\hline $\begin{array}{l}\text { Vivienda a menos de } 250 \mathrm{~m} \text { de un } \\
\text { cauce cloacal a cielo abierto }\end{array}$ & 100.0 & 15 & 0.0 & 0 & 69.4 & 43 & 30.6 & 19 & $\mathrm{a}$ & & & 0.016 \\
\hline $\begin{array}{l}\text { Entra agua en la vivienda cuando } \\
\text { llueve }\end{array}$ & 100.0 & 15 & 0.0 & 0 & 51.6 & 32 & 48.4 & 30 & $\mathrm{a}$ & & & 0.001 \\
\hline $\begin{array}{l}\text { Vivienda a menos de } 50 \mathrm{~m} \text { de una } \\
\text { laguna }\end{array}$ & 40.0 & 6 & 60.0 & 9 & 43.5 & 27 & 56.5 & 35 & 0.864 & 0.274 & 2.725 & 0.083 \\
\hline $\begin{array}{l}\text { Vivienda a menos de } 50 \mathrm{~m} \text { del perí- } \\
\text { metro del vertedero }\end{array}$ & 20.0 & 3 & 80.0 & 12 & 38.7 & 24 & 61.3 & 38 & 0.396 & 0.101 & 1.549 & 0.173 \\
\hline $\begin{array}{l}\text { Problemas en el suministro de } \\
\text { agua corriente }\end{array}$ & 100.0 & 15 & 0.0 & 0 & 93.5 & 58 & 6.5 & 4 & $\mathrm{a}$ & & & 0.581 \\
\hline
\end{tabular}

* Significancia estadística con valores de $p<0.05$.

a. No se calcula, dado que una de las frecuencias observadas es 0 o alguna casilla ha esperado un recuento menor que 5.

\section{Discusión}

$\mathrm{L}$

as enfermedades dermatológicas, particularmente las de carácter infeccioso, suponen en la actualidad un importante problema de salud pública, sobre todo entre poblaciones expuestas a la concurrencia de condiciones socioeconómicas, habitacionales y ambientales adversas; sin embargo, estas no siempre son identificadas y varias de ellas por largo tiempo han sido desatendidas a pesar de que pueden adquirir una alta prevalencia en la comunidad (4,5).

En Paraguay, a diferencia de otros países de la región, son muy escasas las investigaciones respecto a esta problemática en contextos urbanos precarios. Entre los pocos estudios recientes cabe destacar el de Adriana González Freitas, desarrollado en 2011, con población pediátrica que vivía en los alrededores del vertedero de Encarnación, donde se encontró una alta proporción de infantes afectados por problemas de piel (85.7\%) (15). De igual modo, Tatiana Moreno et al., en julio de 2014, estudiaron este mismo tema en un barrio colindante al de nuestro estudio, donde (en coincidencia con nuestros resultados, $79.2 \%$ ) también hallaron una alta prevalencia de lesiones cutáneas infecciosas (74\%) entre los/as niños/as damnificados/as por la inundación del río Paraguay en Asunción (16). El trabajo de Moreno et al., en concordancia con nuestra investigación, relacionó la aparición de dermatosis infecciosas con el desplazamiento a áreas de refugio, donde las familias viven en condiciones de 
espacio más hacinadas y de higiene más precarias. A diferencia de estos dos trabajos que se realizaron en niños, nuestra investigación también reveló una alta presencia de enfermedades dermatológicas en la población joven y adulta mayor de 15 años, los cuales reunieron el $51.9 \%$ de las patologías encontradas.

En América Latina y el resto del mundo, en correspondencia con los resultados de nuestro estudio, varias investigaciones han mostrado también altas prevalencias de dermatosis infecciosas en contextos empobrecidos $(5,17,18)$. Estudios recientes, como el de Raut et al., en Mumbai (India), y el de Maryum et al., en Karachi (Pakistán), arrojaron también altos índices de dermatosis infecciosas en poblaciones urbanas $(6,19)$.

Una prevalencia elevada de micosis superficiales fue encontrada en nuestro estudio (35.1\%), y de ellas las dermatofitosis o tiñas fueron el diagnóstico más frecuente entre todas las patologías (23.4\%), superior a los resultados de trabajos como el de Pal y Pal en un slum de Calcuta (India) en niños/as (26.8\%), o el de El-Khateeb et al., en El Cairo (Egipto) entre población adulta urbana (15.8\%), y de Baur et al., en población adulta de un slum de Calcuta (India) (10.7\%) (20-22). No obstante, prevalencias muy superiores fueron encontradas en el trabajo realizado por Moto et al., en un asentamiento informal de Nairobi (Kenia), donde un $81.1 \%$ de los/as niños/as presentaron dermatofitosis (23). En Paraguay, los estudios de González Freitas y de Moreno et al. mostraron también prevalencias más bajas del $12.6 \%$ y $8 \%$, respectivamente, probablemente debido a que los estudios mencionados fueron desarrollados en niños/as que acudieron a consulta médica en contraste con nuestra investigación, que fue desarrollada prospectivamente mediante visitas domiciliarias $(15,16)$.

Por su parte, Chepchirchir et al. hallaron mayores prevalencias que las nuestras (3.9\%) de tiña de la cabeza en niños/as en el asentamiento urbano de Kiberia (Kenia) (11.2\%) (24). Según estimaciones internacionales, entre el $10 \%$ y el $20 \%$ de la población mundial está afectada por infecciones fúngicas de la piel y se ha apreciado una prevalencia global de onicomicosis del $11 \%$ y porcentajes de entre el $7 \%$ y el $33 \%$ de niños/as afectados/as por tinea capitis evaluados en diversos trabajos clínicos en países del Sur Global (17).

En nuestros resultados, las infecciones bacterianas de la piel estuvieron presentes en el $35.1 \%$ de las personas, en un mayor porcentaje en menores de 15 años (55.6\%). Prevalencias menores documentaron los estudios locales de Paraguay realizados por González Freitas y Moreno et al., con valores del $17.4 \%$ y $16 \%$, respectivamente $(15,16)$. Del mismo modo, se observaron porcentajes más bajos en los estudios de Maryum et al., en Karachi (Pakistán), entre población adulta (10\%) o en el de Pal y Pal en escuelas de Calcuta (India) (9.26\%) y Baur et al., en un slum de la misma ciudad (8.9\%) (19,20,22). En la línea de estos trabajos, Bowen et al., en una revisión global de investigaciones epidemiológicas realizadas entre 1970 y 2014 sobre impétigo y pioderma, concluyeron que la población pediátrica con mayores prevalencias se encuentra principalmente en contextos tropicales de escasos recursos (25). 
En contraste con los resultados de otros trabajos, en nuestro estudio, la forunculosis destacó como una de las patologías infecciosas más prevalentes, hallada en un $19.5 \%$ de las personas examinadas. Porcentajes mucho menores fueron hallados en los trabajos de El-Khateeb et al., en el Cairo (3\%), y por Baur et al., en Calcuta (India) $(1.3 \%)(21,22)$.

En nuestro estudio, la escabiosis evidenció también una prevalencia importante (15.6\%). Prevalencias algo mayores (19.8\%) las hallaron en un slum de Beira (Mozambique) Chhaganlal et al. (26). En una reciente revisión de investigaciones de May et al. se estimó que la escabiosis (a excepción de Europa y Oriente Medio) afecta a más del $10 \%$ de la población en diferentes regiones del mundo, y que la población pediátrica que vive en entornos de recursos limitados es la más afectada (17). Por su parte, Romani et al. concluyeron también en una revisión de publicaciones que las mayores prevalencias de escabiosis en el mundo concurren, sobre todo, en las regiones del Pacífico y América Latina, sustancialmente más en niños que en adolescentes y adultos. En contraste con este trabajo, en nuestro estudio se manifestó una prevalencia similar tanto en niños como en jóvenes y adultos de 15 y más años (50\%) (18).

En la región, porcentajes menores a nuestros resultados se encontraron en un estudio realizado por Heukelbach et al. en una favela de la ciudad de Fortaleza (Brasil), con una prevalencia del $8.8 \%$ (7). En el ámbito local, en Paraguay, los estudios de González Freitas y de Moreno et al. también encontraron porcentajes menores de un $1.58 \%$ y un $8 \%$, respectivamente $(15,16)$. La mayor prevalencia encontrada en nuestro estudio se podría deber a que la escabiosis no es motivo de consulta médica recurrente; mientras que nuestra identificación de la enfermedad fue realizada en visitas sobre terreno.

Por otro lado, May et al. y Salazar han destacado los climas cálidos, especialmente los entornos húmedos (sub)tropicales, como el que caracteriza a la ciudad de Asunción (con una media anual de $25^{\circ} \mathrm{C}$ y máximas temperaturas entre diciembre y marzo que rebasan los $30^{\circ} \mathrm{C}$ en horas de mayor exposición solar) (27), como más susceptibles al desarrollo de enfermedades de la piel de carácter infeccioso, al igual que en nuestros resultados, fundamentalmente para micosis superficiales o pioderma $(17,28)$. Al efecto del clima se suman otras características geológicas particulares proclives a propiciar ambientes húmedos, como la ubicación de las viviendas en áreas próximas a cursos hídricos (29). La orografía del territorio de nuestro estudio, ubicado en la ribera del río Paraguay, en un área de antiguos humedales, atravesado por cuatro arroyos y con la presencia de varias lagunas internas, propicia que las viviendas de las personas examinadas estén situadas (en gradientes diferenciales) en un ambiente altamente húmedo por su proximidad a estos (11).

Varios estudios han relacionado las infecciones cutáneas bacterianas con los problemas de provisión de agua corriente. En este sentido, las dificultades o falta de acceso a agua limpia y las deficitarias prácticas de higiene derivadas de la carencia de esta en los hogares están estrechamente implicadas en favorecer enfermedades infecciosas de la piel; por ello, 
la cantidad individual y calidad del agua para el aseo corporal y del entorno constituyen factores importantes a considerar $(8,30)$.

Adicionalmente, trabajos como los de Bowen et al., Yeoh et al. y Costa et al. han observado que las altas prevalencias de impétigo o pioderma están estrechamente correlacionadas con antecedentes de escabiosis o tiñas en contextos de baja higiene $(25,31,32)$. De igual modo, según Ibler y Kromann, las dermatitis o eccemas, heridas o úlceras, sumadas a escaso aseo corporal, aumentan la susceptibilidad de colonización bacteriana, sobre todo a Streptococcus pyogenes y Staphylococcus aureus (33). Las lesiones de la piel primarias crean posibles portales de entrada a bacterias patógenas que si no son bien tratadas, pueden derivar en infecciones con una morbilidad secundaria de mayor gravedad y que pueden evolucionar en afecciones renales y cardiacas potencialmente severas y crónicas (31). Es por ello que estos autores recomiendan no banalizar como meras irritaciones de piel estas lesiones cutáneas, ya que un mal cuidado de éstas conllevaría un importante impacto para la salud de las personas $(5,18)$.

En correspondencia con las condiciones de los hogares de las personas examinadas en nuestro estudio, donde la relación de convivientes por dormitorio fue alta, varias investigaciones han señalado que la transmisión interpersonal, especialmente en los casos de pioderma, escabiosis o tiñas, está bien establecida como condición de contagio y difusión de la enfermedad en el entorno íntimo y próximo $(29,34)$. Las prácticas sociales que involucran un contacto piel con piel, favorecidas por el número de personas que comparten cama, situación más comúnmente frecuente en hogares de escasos recursos, puede contribuir ostensiblemente a la proliferación de estas enfermedades dermatológicas infecciosas.

Más recientemente, un trabajo sobre condiciones que influyen en la prevalencia de pioderma desarrollado en Indonesia por Indria Depari et al. señaló igualmente que una alta densidad de convivientes en el hogar, particularmente por dormitorio, facilita las posibilidades de colonización, infección, transmisión y sobreinfección por Staphylococcus aureus (35). En este mismo sentido, Ibler y Kromann plantean la necesidad de investigar más sobre las formas y calidad de vida de las personas afectadas (específicamente en su caso por forunculosis recurrente) para ampliar el conocimiento sobre las vías de transmisión, con la intención de promover modos de prevención en infecciones de la piel o tejidos blandos. Estos mismos autores proponen insistir en una combinación de medidas de carácter individual y de higiene en el hogar y en el entorno comunitario. Las primeras enfocadas en información educativa promoción sobre higiene personal y, a su vez, sugieren pautas de desinfección en el hogar y entorno comunitario mediante la limpieza de superficies con las que se tiene habitual contacto (33).

Por otro lado, en concordancia con el contexto de nuestro estudio, donde se produce una importante convivencia con animales escasamente vigilados sanitariamente: perros (66.2\%), cerdos y gallinas ( $46.8 \%$ ), fundamentalmente, de acuerdo con los trabajos de Feldmeier y Heukelbach y Feldmeier et al., realizados en Brasil, el contacto humano-animal-tierra es 
considerado un factor que favorece las enfermedades infecciosas de la piel $(36,37)$. Estos autores señalan, en el caso particular de las ectoparasitosis, a los animales como uno de los principales reservorios que aseguran la trasmisión permanente, que facilitan los modos de propagación de agentes infecciosos y que aumentan las posibilidades de contagio. Ello nos hace pensar acerca de la necesidad de una vigilancia sanitaria mayor sobre los animales del territorio del estudio. Así mismo, el contacto del humano con arenas infectadas por excretas humanas o animales se indica también como favorecedor de infecciones cutáneas por varios tipos de hongos, parásitos o bacterias presentes, en especial si los pies no están protegidos con calzado. En concordancia con las condiciones del terreno y habitabilidad del estudio, estos mismos autores indican que entornos urbanos, como el de nuestro estudio, donde las calles no están pavimentadas, hay presencia de desechos y excretas esparcidos en las cercanías de las viviendas y prima la presencia de arena o barro, son predisponentes a la proliferación de ectoparásitos como la tunga penetrans o la larva migratoria $(36,38)$.

Por su parte, Riley et al. y Ehrenberg y Ault señalan también que las desigualdades urbanas se manifiestan de diferentes formas a través de la piel y afirman que particularmente las ectoparasitosis suponen un buen marcador de las inequidades en las ciudades $(4,5)$. Para estos autores, la urbanización desigual en muchos países del Sur Global ha generado condiciones diferenciales que facilitan su proliferación entre la población de los territorios urbanos más anegados y desatendidos, donde a menudo se concentran los sectores sociales de menores ingresos. Es por ello que señalan la alta prevalencia de enfermedades parasitarias como uno de los principales indicadores de abandono de las políticas de salud pública, y de la sociedad en general, dado que adquieren una realidad más severa en los territorios más desfavorecidos (5).

En nuestro estudio, otra expresión recurrente de estas desigualdades urbanas en el territorio toma forma en la presencia constante de efluentes cloacales y pluviales que descargan sus desechos en los arroyos que lo atraviesan, arroyos que además reciben habitualmente gran cantidad de residuos sólidos a lo largo de su curso de agua. El escaso e inadecuado sistema pluvial y un irregular sistema de alcantarillado urbano en la ciudad tiene como consecuencia en los Bañados de Asunción la inundación de patios y viviendas (diferencialmente) por aguas cloacales de raudal provenientes del desborde de los arroyos en esta área de la ciudad los días de intensas y recurrentes lluvias subtropicales (39). Diferentes estudios han identificado la manifestación de enfermedades infecciosas de la piel en relación con el contacto directo con aguas residuales (40).

De igual modo, en los últimos años se ha apuntado que los asentamientos urbanos informales pueden ser considerados puntos críticos para la transmisión ambiental de la resistencia antimicrobiana por la alta densidad de humanos, animales y alimañas; por el uso frecuente de antibióticos como consecuencia de una mayor recurrencia de patologías y 
automedicación, y especialmente por la deficiente infraestructura de agua potable, drenaje, saneamiento y recolección de residuos (41).

A los factores de carácter socioambiental ya mencionados, se suman otros, señalados por varios autores, que influyen en la morbilidad y la evolución de las enfermedades dermatológicas de carácter infeccioso, como son las condiciones de los dispositivos de atención a la salud existentes y los patrones de búsqueda de consulta e identificación ante ciertas lesiones cutáneas en contextos de precariedad y desprotección social en salud $(5,36)$.

En nuestro trabajo, un porcentaje relativamente alto de personas (77.9\%) no había recurrido a consulta médica por lesiones cutáneas. Según Salazar, los motivos que explican este patrón de búsqueda de atención de salud corresponden principalmente a que el cuadro remite espontáneamente, a que se opta por la automedicación, a que se recurre a sistemas médicos alternativos o a limitaciones de acceso por la ubicación geográfica o por su condición de no asegurados $(28,42)$.

En este mismo sentido, los trabajos de Ehrenberg y Ault y de El-Khateeb et al. concluyen que los estigmas socioculturales sobre algunas enfermedades dermatológicas también influyen en el ocultamiento de algunas de ellas $(5,43)$. El temor a ser rechazados por ser considerados fuente de contagio; el desconocimiento e indiferencia sobre el agravamiento que pueden contraer ciertas lesiones cutáneas, debido a la obligación de mantener la actividad de subsistencia laboral, o la falta de acceso a chequeos regulares tanto en infantes como en adultos por los dispositivos de atención, tiene como resultado, según los autores, la generación de una "comunidad de pacientes silenciosos" que no se hace presente en la consulta médica. En coincidencia con estos y otros autores, en nuestro trabajo hemos observado un efecto de normalización, en el caso particular de las ectoparasitosis, micosis y forunculosis, que pasan a menudo desapercibidas para la persona afectada e incluso para el personal sanitario local, por ser muy comunes en el medio, con frecuencia resueltas en el entorno doméstico de la persona, recurriendo habitualmente a la autoatención con remedios caseros y naturales $(31,42,44)$.

En Paraguay, al igual que en otros países de la región, las enfermedades dermatológicas, a pesar de la magnitud que ha ido adquiriendo esta problemática, están subdiagnosticadas o las evidencias de estudios localizados, tanto en el ámbito rural como en el urbano, son aún muy escasas. Debido a esta ausencia, en Paraguay hoy en día es difícil conocer de forma precisa la realidad e impacto de las enfermedades dermatológicas, especialmente las de carácter infeccioso, que son las más preocupantes, por su morbilidad primaria y secundaria, y su potencial incidencia en las condiciones de vida de los habitantes de los asentamientos urbanos precarios donde confluyen diversas condiciones de exposición a contraer enfermedades dermatológicas.

Las limitaciones de este estudio refieren a la disposición de una pequeña muestra. En este sentido, nos parece importante posibilitar futuros análisis que profundicen sobre las posibles 
relaciones trazadas en este trabajo entre condiciones sociales, habitacionales, ambientales y enfermedades dermatológicas a partir de estudios más amplios y comparativos que tengan en cuenta las desigualdades territoriales urbanas. A su vez, para una mejor caracterización y manejo de las enfermedades infecciosas cutáneas y condiciones ambientales sería oportuno realizar pruebas microbiológicas que permitan conocer los microrganismos y los patógenos involucrados $(38,41)$.

Como principales hallazgos de este estudio, los resultados sugieren, de acuerdo con la literatura revisada, que la alta prevalencia de enfermedades cutáneas infecciosas podría relacionarse con una mayor exposición a condiciones socioambientales locales adversas. Un clima subtropical de altas temperaturas y húmedo, una intensa congregación de convivientes en el hogar, problemas de provisión de agua, presencia de suelos de arena y animales con escasa vigilancia sanitaria, así como un sistema de evacuación de excretas deficiente, la proximidad a arroyos y cauces al aire libre depositarios de materia cloacal y el impacto recurrente de raudales los días de intensa lluvia, unidas a las dificultades de acceso y resolución de los dispositivos de atención a la salud, podrían ser considerados factores relevantes para mejorar las políticas públicas de salud de las enfermedades dermatológicas en la población de asentamientos urbanos precarios.

Desde la determinación social, ambiental y ecosindémica, especialmente en contextos empobrecidos urbanos, estas condiciones analizadas, en su acción sinérgica e interrelacionada, propician un hábitat favorecedor de altas prevalencias dermatológicas, tanto en población infantil como en adulta, que son expresión de la incorporación social de la enfermedad y del impacto de las desigualdades urbanas sobre la salud de los habitantes que viven en los territorios más deteriorados y desatendidos de la ciudad, como son los asentamientos urbanos precarios (45-47).

En esta misma dirección, se considera importante la implementación de políticas en salud pública que tengan en cuenta la heterogeneidad y las microdiferencias de los territorios y que posibiliten un mayor conocimiento, prevención y atención de las enfermedades infecciosas de la piel en poblaciones en contextos que conjugan múltiples condiciones de exposición $(2,11)$. Ello contribuiría a comprender mejor la morbilidad primaria y secundaria asociada a estas y las estrategias a las que recurre una numerosa "comunidad de pacientes silenciosos" que no acuden a la consulta médica.

\section{Agradecimientos}

$A_{\text {sur. }}^{\text {todos los participantes de este estudio, por su colaboración, y a la comunidad de Bañado }}$ 


\section{Contribución de los autores}

Todos los autores han contribuido de igual forma a las etapas de preparación de este texto; asimismo, dieron su aprobación final y aceptan ser responsables de todos los aspectos del trabajo.

\section{Financiación}

Esta investigación se realizó en el marco del proyecto “Salud, enfermedad y pobreza urbana: de Asunción” (14-INV-236), desde el Centro de Documentación y Estudios, financiado por el programa Prociencia del Consejo Nacional de Ciencia y Tecnología (Conacyt) del Paraguay.

\section{Conflicto de intereses}

$\mathrm{N}^{\text {nesuroudectarata }}$

\section{Referencias}

1. Ezeh A, Oyebode O, Satterthwaite D, Chen YF, Ndugwa R, Sartori J, et al. The history, geography, and sociology of slums and the health problems of people who live in slums. The Lancet. 2017;389(10068):547-58. https://doi.org/10.1016/S0140-6736(16)31650-6

2. Lilford RJ, Oyebode O, Satterthwaite D, Meléndez-Torres GJ, Chen YF, Mberu B, et al. Improving the health and welfare of people who live in slums. Lancet. 2017;389(10068):55970. https://doi.org/10.1016/S0140-6736(16)31848-7

3. Unger A. Children’s health in slum settings. Arch Dis Child. 2013;98(10):799-805. https:// doi.org/10.1136/archdischild-2011-301621

4. Riley LW, Ko AI, Unger A, Reis MG. Slum health: diseases of neglected populations. BMC Int Health Hum Rights. 2007;7:2. https://doi.org/10.1186/1472-698X-7-2

5. Ehrenberg J, Ault S. Neglected diseases of neglected populations: thinking to reshape the determinants of health in Latin America and the Caribbean. Biomedcentral Public Health. 2005;5(119):1-13. https://doi.org/10.1186/1471-2458-5-119

6. Raut AV, Pakhare AP, Suryawanshi SR. Morbidity profile and health seeking behaviour among youth - a cross-sectional study from a slum in Mumbai. Online J Health Allied Scs. 2017;16(3):2. 
7. Heukelbach J, Van Haeff E, Rump B, Wilcke T, Moura RC, Feldmeier H. Parasitic skin diseases: health care-seeking in a slum in north-east Brazil. Trop Med Int Health. 2003;8(4):368-73. https://doi.org/10.1046/j.1365-3156.2003.01038.x

8. Plaza G del C, Zapata AO. Residuos y salud: Tartagal-Salta. RECyT [internet]. 2011;16(1):35-43.

9. Dirección General de Estadística, Encuestas y Censos (DGEEC). Estratificación socioeconómica por segmentos: Censo Nacional de Población y Viviendas, 2012 [internet]. Fernando de la Mora (Paraguay): DGEEc; 2018. Disponible en: https://www.ine.gov.py/Publicaciones/ Biblioteca/ESS-CNVP2012/Estratificacion\%20Socioeconomica\%20de\%20Segmentos\%20 CNPV\%202012.pdf

10. Dirección General de Estadística, Encuestas y Censos (DGEEC). Necesidades básicas insatisfechas (NBI): una aproximación a la pobreza estructural, 2012 [internet]. Fernando de la Mora (Paraguay): DGEec. 2016. Disponible en: https://www.ine.gov.py/Publicaciones/ Biblioteca/documento/ebc6_Necesidades\%20Basicas\%20Insatisfechas\%20(NBI).pdf

11. Monte Domecq R, Báez J, Ávila JL. Las inundaciones en el Paraguay. Rev Fac Cienc Tecnol (Asunción). 2016;7:22-40.

12. World Medical Association. Declaration of Helsinki. Ethical principles for medical research involving human subjects [internet]. 2001. Disponible en: https://www. who.int/ bulletin/archives/79\%284\%29373.pdf

13. American Anthropological Association (AAA). Principles of professional responsibility [internet]. 2012. Disponible en: http://ethics.americananthro.org/category/statement/

14. Associação Brasileira de Antropologia ( $\mathrm{ABA}$ ). Código de Ética do Antropólogo e da Antropóloga [internet]. 2012. Disponible en: http://www.portal.abant.org.br/index.php/ institucional/codigo-de-etica

15. González Freitas A. Impacto de las condiciones socioeconómicas y ambientales con relación a la presencia de enfermedades dermatológicas en la población infantil en el barrio San Antonio Ypecurú (vertedero municipal). Revista sobre Estudios e Investigaciones del Saber Académico. 2011;5:73-77.

16. Moreno T, Rodríguez L, Salgueiro L, Riveros R, Mancía S, Narváez D, et al. Patologías cutáneas en niños que habitan en refugios de zonas inundadas. Rev Pediatr (Asunción). 2016;43(1):39-44. https://doi.org/10.18004/ped.2016.abril.39-44

17. May P, Bowen A, Tong S, Steer A, Prince S, Andrews R, Currie B, Carapetis J. Protocol for the systematic review of the prevention, treatment and public health management of impetigo, scabies and fungal skin infections in resource-limited settings. Syst Rev. 2016;5(1):162. https://doi.org/10.1186/s13643-016-0335-0

18. Romani L, Steer AC, Whitfeld MJ, Kaldor JM. Prevalence of scabies and impetigo worldwide: a systematic review. Lancet Infect Dis. 2015;15(8):960-7. https://doi.org/10.1016/ S1473-3099(15)00132-2

19. Maryum H, Alam MZ, Ahmed I. Pattern of skin diseases in a tertiary care private hospital, Karachi. J Pak Assoc Dermatol. 2014;24:292-7.

20. Pal J, Pal AK. Impact of health education regarding personal hygiene and dietary habits on morbidity profile of students: an intervention study in a government secondary school in a slum area of Kolkata. Int J Community Med Public Health. 2017;4:2492-7. https://doi.org/10.18203/2394-6040.ijcmph20172846 
21. El-Khateeb EA, Imam AA, Sallam MA. Pattern of skin diseases in cairo, Egypt. Int J Dermatol. 2011;50:844-853. https://doi.org/10.1111/j.1365-4632.2010.04840.x

22. Baur B, Sarkar J, Manna N, Bandyopadhyay L. The pattern of dermatological disorders among patients attending the skin OPD of a tertiary care hospital in Kolkata, India. J Dent Med Sci. 2013;4:4-9. https://doi.org/10.9790/0853-0340409

23. Moto JN, Maingi JM, Nyamache AK. Prevalence of Tinea capitis in school going children from Mathare, informal settlement in Nairobi, Kenya. BMC Res Notes. 2015;8:274. https:// doi.org/10.1186/s13104-015-1240-7

24. Chepchirchir A, Bii C, Ndinya-Achola J.O. Dermatophyte infections in primary school children in Kibera slums of Nairobi. East Afr Med J. 2009;86:59-68. https://doi.org/10.4314/ eamj.v86i2.46934

25. Bowen AC, Mahe A, Hay RJ, Andrews RM, Steer AC, Tong SY et al. The Global Epidemiology of Impetigo: a systematic review of the population prevalence of impetigo and pyoderma. PLoS One. 2015;10(8). https://doi.org/10.1371/journal.pone.0136789

26. Chhaganlal K, Van Jaarsveld I, Hoffmann K, Ramos MI, Krober M, De Hoop, D. Cutaneous disorders in the "bairro Inhamudima" of Beira, Mozambique. Int J Dermatol. 2007;46(2):358. https://doi.org/10.1111/j.1365-4632.2007.03482.x

27. Itaipu Binacional. Atlas del potencial energético solar y eólico del Paraguay [internet]. Asunción: Itaipu Binacional-Parque Tecnológico de Itaipu; 2016. Disponible en: https:// pese.pti.org.py/static/geonode/img/atlas.pdf

28. Salazar K. Situación de la forunculosis en las poblaciones de Iroquois, Mercedes, La Argentina, Milano y Tierra Grande del Cantón de Guácimo y Siquirres de Limón, Costa Rica. Dermatol Rev Mex. 2013;57(6):438-45.

29. World Health Organization. Epidemiology and management of common skin diseases in children in developing countries [internet]. Geneva (Switzerland); 2005. Disponible en: https://apps.who.int/iris/bitstream/handle/10665/69229/WHO_FCH_CAH_05.12_eng. pdf?sequence=1\&isAllowed $=y$

30. Hazarika AN. Clinico-epidemiological study of pyoderma in children. Nat J Res Com Med. 2012;1(4):178-241. http://doi.org/10.18231/2581-4761.2019.0014

31. Yeoh DK, Anderson A, Cleland G, Bowen AC. Are scabies and impétigo "normalised"? A cross-section al comparative study of hospitalised children in northern Australia assessing clinical recognition and treatment of skin infections. PLoS Negl Trop Dis. 2017;11(7):e0005726. https://doi.org/10.1371/journal.pntd.0005726

32. Costa ML, Rodríguez Heredia O, García Perera A, Rodríguez Escobar N. Aspectos epidemiológicos de la escabiosis infantil en el municipio Cabimas, estado Zulia, Venezuela. Archivo Médico de Camagüey. 2008;12(2).

33. Ibler KS, Kromann CB. Recurrent furunculosis - challenges and management: a review. Clin Cosmet Investig Dermatol. 2014;7:59-64. https://doi.org/10.2147/CCID.S35302

34. Beltrán MA, García del Corro HJ, Couso M, Gallo MD, Lettieri A, Barna PV. Relación entre hacinamiento e infecciones comunitarias de piel y partes blandas. Medicina (Buenos Aires). 2017;77(6):465-8. 
35. Indria Depari L, Sugiri U, Ilona Fuad Abdul Hamied L. Relation between risk factors of pyoderma and pyoderma incidence. Althea Med J. 2016;3(3):434-9. https://doi. org/10.15850/amj.v3n3.867

36. Feldmeier H, Heukelbach J. Epidermal parasitic skin diseases: a neglected category of poverty-associated plagues. Bull World Health Organ. 2009;87(2):152-9. https://doi. org/10.2471/BLT.07.047308

37. Feldmeier H, Heukelbach J, Ugbomoiko US, Sentongo E, Mbabazi P, et al. Tungiasis, a neglected disease with many challenges for global public health. PLoS Negl Trop Dis. 2014;8(10):e3133. https://doi.org/10.1371/journal.pntd.0003133

38. Junior VH, Mendes AL, Talhari CC, Miot HA. Impact of environmental changes on Dermatology. An Bras Dermatol. 2021;96(2):210-23. https://doi.org/10.1016/j. abd.2020.11.004

39. Rivelli E, Báez J, Monte Domecq R. Identificación de puntos críticos a inundaciones urbanas en la ciudad de Asunción. Rev Fac Cienc Tecnol(Asunción). 2014;1:46-53.

40. Balato N, Megna M, Ayala F, Balato A, Napolitano M, Patruno C. Effects of climate changes on skin diseases. Expert Rev Anti Infect Ther. 2014;12:171-81. https://doi.org/10.158 6/14787210.2014.875855

41. Nadimpalli ML, Marks SJ, Montealegre MC, Gilman RH, Pajuelo MJ, Saito M, et al. Urban informal settlements as hotspots of antimicrobial resistance and the need to curb environmental transmission. Nat Microbiol. 2020;5:787-95. https://doi.org/10.1038/s41564020-0722-0

42. Rodero P, Merino I, coordinadores. Salud, enfermedad y pobreza urbana: estudio de los procesos de salud, enfermedad y atención de las familias en la periferia sur de Asunción [internet]. Asunción: CDE-Prociencia conacyt Paraguay; 2018. Disponible en: https://www. conacyt.gov.py/sites/default/files/upload_editores/u489/salud-enfermedad-pobreza-urbana-libro.pdf

43. El-Khateeb EA, Lotfi RA, Abd Elaziz KM, El-Shiekh SE. Prevalences of skin diseases among primary schoolchildren in Damietta, Egypt. Int J Dermatol. 2014;53(5):609-16. https:// doi.org/10.1111/ijd.12335

44. Worth C, Heukelbach J, Fengler G, Walter B, Lisenfeld O, Feldmeier H. Impaired quality of life in adults and children with scabies from an impoverished community in Brazil. Int J Dermatol. 2012;51:275-82. https://doi.org/10.1111/j.1365-4632.2011.05017.x

45. Krieger N. Theories for social epidemiology in the 21st century: an ecosocial perspective. Int J Epidemiol. 2001;30(4):668-77. https://doi.org/https://doi.org/10.1093/ije/30.4.668

46. Breilh J. La determinación social de la salud como herramienta de transformación hacia una nueva salud pública (salud colectiva). Rev Fac Nac Salud Pública. 2013;31(suppl 1):13-27.

47. Singer M, Bulled N, Ostrach B, Mendenhall E. Syndemics and the biosocial conception of health. Lancet. 2017;389(10072):941-50. https://doi.org/10.1016/S0140-6736(17)30003-X 